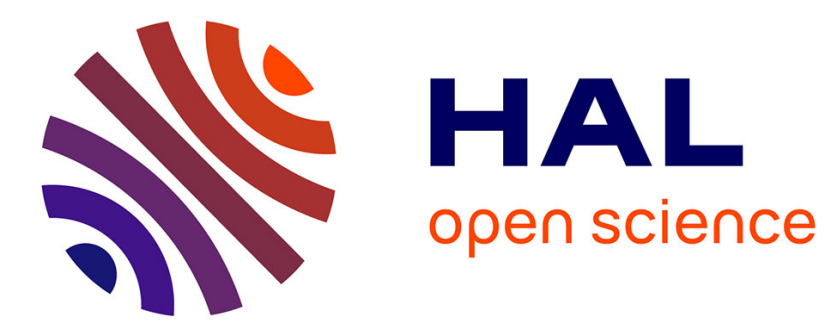

\title{
Le cinéma de Luis Estrada ou le passage de la nécropolitique à la nécroesthétique \\ Davy Desmas
}

\section{To cite this version:}

Davy Desmas. Le cinéma de Luis Estrada ou le passage de la nécropolitique à la nécroesthétique. Amerika - Mémoires, identités, territoires, 2015, 12, 10.4000/amerika.6580 . hal-01812558

\section{HAL Id: hal-01812558 \\ https://hal.science/hal-01812558}

Submitted on 11 Jun 2018

HAL is a multi-disciplinary open access archive for the deposit and dissemination of scientific research documents, whether they are published or not. The documents may come from teaching and research institutions in France or abroad, or from public or private research centers.
L'archive ouverte pluridisciplinaire HAL, est destinée au dépôt et à la diffusion de documents scientifiques de niveau recherche, publiés ou non, émanant des établissements d'enseignement et de recherche français ou étrangers, des laboratoires publics ou privés. 


\section{Amerika Amerika \\ Mémoires, identités, territoires}

12 | 2015

La mort : imaginaires et sociétés

\section{Le cinéma de Luis Estrada ou le passage de la nécropolitique à la nécroesthétique}

\section{Davy Desmas}

\section{(2) OpenEdition}

1 Journals

\section{Édition électronique}

URL : http://journals.openedition.org/amerika/6580

DOI : 10.4000/amerika.6580

ISSN : 2107-0806

\section{Éditeur}

LIRA-Université de Rennes 2

Ce document vous est offert par Université Fédérale Toulouse Midi-Pyrénées

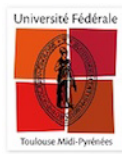

\section{Référence électronique}

Davy Desmas, « Le cinéma de Luis Estrada ou le passage de la nécropolitique à la nécroesthétique », Amerika [En ligne], 12 | 2015, mis en ligne le 01 juillet 2015, consulté le 11 juin 2018. URL : http:// journals.openedition.org/amerika/6580; DOI : 10.4000/amerika.6580

Ce document a été généré automatiquement le 11 juin 2018.

(c) Tous droits réservés 


\title{
Le cinéma de Luis Estrada ou le passage de la nécropolitique à la nécroesthétique
}

\author{
Davy Desmas
}

1 La présente réflexion s'inscrit dans le champ des interrogations récemment soulevées par l'auteure mexicaine Cristina Rivera Garza à propos de la nécessité pour les artistes de dire l'horreur dans laquelle est plongé le Mexique depuis quelques décennies, et tout particulièrement depuis le passage au XXI ${ }^{\mathrm{e}}$ siècle. Dans des œuvres comme Dolerse. Textos desde un país herido (2011) et Los muertos indóciles. Necroescrituras y desapropiación (2013), elle écrit :

Lo que los mexicanos de inicios del siglo XXI hemos sido obligados a ver - ya en las calles, en los puentes peatonales, en la televisión o en los periódicos - es, sin duda, uno de los espectáculos más escalofriantes del horrorismo contemporáneo. Los cuerpos abiertos en canal, vueltos pedazos irreconocibles sobre las calles. Los cuerpos extraídos en estado de putrefacción de cientos y cientos de fosas. Los cuerpos sin manos o sin orejas o sin narices; los cuerpos ya sin aire; los cuerpos sin voz. (Rivera Garza, $2011: 8$ )

2 Elle ajoute quelques lignes plus loin:

Cuando todo enmudece, cuando la gravedad de los hechos rebasa con mucho nuestro entendimiento e incluso nuestra imaginación, entonces está ahí, dispuesto, abierto, tartamudo, herido, balbuceante, el lenguaje del dolor. De ahí la urgencia estética de decir, en el más básico y también en el más desencajado de los lenguajes, esto me duele. (ibid. : 9)

Selon Rivera Garza, c'est cette nécessité de crier l'horreur et la douleur qui a provoqué, depuis le milieu des années 1990, l'apparition salutaire d'une nécroécriture, un concept que nous élargirons à la nécroesthétique dans le cadre de ce travail, centré sur le cinéma. Par ailleurs, le questionnement sur la participation des autorités ou du pouvoir à la généralisation de la mort se fondera sur les réflexions lancées par Michel Foucault et Giorgio Agamben à propos du biopouvoir, c'est-à-dire la prise en compte de la vie (et donc de la mort) par le pouvoir, au sein d'un Etat moderne qui est passé d'une logique consistant à «faire mourir et laisser vivre » à la logique inverse, autrement dit «faire vivre et laisser mourir » (Foucault, 1997 : 214). 
Luis Estrada est indéniablement de ces artistes qui interrogent la place de la mort et de l'horreur dans la société mexicaine contemporaine. Auteur d'une œuvre sulfureuse, évoluant sur une corde raide l'ayant amené à subir à plusieurs reprises les foudres de la censure, Luis Estrada s'attache à éclairer les rouages du pouvoir et les vices de ses détenteurs, le tout dans le contexte du Mexique actuel. Les deux œuvres sur lesquelles va porter la réflexion s'inscrivent dans le genre de la comédie. Le premier film, La ley de Herodes, qui est sorti sur les écrans en 1999, soit à la veille des élections présidentielles, retrace le parcours de Juan Vargas, à qui l'on propose de devenir maire d'un petit village. Alors que Vargas voyait là une formidable opportunité de gravir les échelons de son parti, le Parti Révolutionnaire Institutionnel (PRI), il fait la difficile expérience de l'exercice du pouvoir, dans un village totalement isolé et dépourvu de fonds publics en raison des exactions du précédent maire. Alors qu'il souhaite démissionner, son chef l'oblige à garder son poste, en l'incitant à se faire respecter et à user de la force pour obtenir de l'argent des habitants du village. Vargas découvre alors l'ivresse du pouvoir, en appliquant les lois à sa guise et en amassant des sommes considérables d'argent. La date de sortie en salle du second film analysé, intitulé El infierno, est tout aussi polémique : le film fait son apparition en septembre 2010, soit exactement deux siècles après le lancement du processus indépendantiste mexicain. Le statut du film est très particulier, puisqu'il répond à un appel d'offres lancé par l'une des plus grandes institutions culturelles du pays, l'IMCINE (Instituto Mexicano de Cinematografía), lui-même lié au pouvoir en place, appel d'offres qui devait permettre à différents réalisateurs de participer aux cérémonies officielles de célébration du bicentenaire. L'intrigue du film est pourtant bien loin d'adopter une vision consensuelle du pays qu'il est censé célébrer : $E l$ infierno retrace l'itinéraire de Benjamín García, alias El Benny, qui regagne son village natal au Mexique après vingt ans passés aux États-Unis. Après avoir tenté dans un premier temps de ne pas céder aux sirènes de l'argent facile, dans une région où le seul secteur prospère est le trafic de drogue, Benjamín finit par se compromettre et intégrer l'univers du narcotrafic, afin d'apporter une aide financière à la veuve de son frère, mort assassiné, et à son neveu.

5 Un des principaux éléments nous autorisant à ériger ces deux films en objets artistiques à même de questionner le Mexique contemporain serait l'effet spéculaire qui s'établit entre les deux vill(ag)es représentées à l'écran et le pays dans sa totalité. Tant San Pedro de los Aguaros, dans La ley de Herodes, que San Miguel Arcángel, dans El infierno, fonctionnent à la manière de microcosmes reproduisant à moindre échelle les logiques politiques, sociales et économiques qui prévalent dans le Mexique moderne. Cela est particulièrement net dans La ley de Herodes : l'interminable succession de maires membres du PRI fait immédiatement de San Pedro de los Aguaros le reflet d'un pays qui a été pendant plus de soixante-dix ans aux mains du même parti. De même, le personnage du docteur Morales, principal opposant au héros car membre du Parti Action Nationale (PAN), reproduit l'hypocrisie morale dont est fréquemment taxé le PAN : effectivement, alors qu'il s'érige en défenseur des valeurs citoyennes et catholiques, taxant le PRI de népotisme et d'immoralité ${ }^{1}$, le spectateur découvre finalement que Morales viole la jeune fille qui travaille comme domestique sous son toit. De son côté, l'influence dont dispose le curé de San Pedro rappelle la position qui est celle de l'Eglise catholique à l'échelle nationale, notamment dans ses liens avec le pouvoir. Dans le film, le prêtre joue sur deux tableaux, puisqu'il extorque de l'argent aux habitants du village en échange de l'absolution de leurs péchés, tout en revendant à prix d'or les confessions de ces mêmes 
habitants à Vargas, le maire, qui peut alors en tirer profit pour empêcher ses ennemis de lui nuire. Le personnage savoureux de Doña Lupe, la mère maquerelle, incarne quant à lui la collusion qui s'établit entre le pouvoir politique et la criminalité, puisque le maire accepte de fermer les yeux sur son commerce, en échange d'une grasse rétribution.

La relation entre Vargas et Robert Smith, enfin, ne peut manquer d'évoquer les relations ambiguës entre le Mexique et les États-Unis : la rencontre entre le maire mexicain et l'ingénieur Smith, alors que Vargas vient de tomber en panne sur le bord d'une route, est d'emblée placée sous le signe de l'hypocrisie; tandis que Robert lui ment en rebranchant un simple fil et en exigeant de lui une somme d'argent considérable, Vargas lui demande un délai et transforme le nom du village dont il est maire en lui disant : "Te vas a San Juan de los Camotes, y allá me esperas sentado ». Au-delà de l'allusion au simple tubercule, c'est ici à la symbolique phallique du camote que renvoie l'albur de Vargas, et donc à l'établissement d'un rapport de forces visant à déterminer qui, selon les mots de Vargas, « es el más chingón ». Le fil de leurs relations, qui se terminent après que le héros a découvert Robert au lit avec sa propre femme, et que cette dernière a finalement décidé de fuir aux États-Unis avec l'ingénieur en emportant l'argent de Vargas, semble ainsi métaphoriser les rapports conflictuels qu'a toujours entretenus le Mexique avec le grand voisin du Nord, celui-ci sortant systématiquement gagnant de ses relations avec l'Amérique Latine, notamment lorsqu'il est question d'une dette à rembourser. C'est cette même vision caustique des relations mexicano-états-uniennes que l'on retrouve lors d'un dîner organisé par Vargas en présence de tous les notables de San Miguel, au cours duquel Smith est interrogé sur l'impact que la nationalisation du pétrole lancée par Lázaro Cárdenas a eu sur l'économie états-unienne ; non sans fanfaronner, Smith répond alors que son pays n'est pas inquiet, et que, comme toujours, il récupèrera bien plus que ce qu'il a perdu... Le même effet spéculaire est présent dans $E l$ infierno, dans lequel on retrouve certaines des figures qui peuplaient déjà San Pedro de los Aguaros, cette fois transposées au monde du narcotrafic: le maire crapuleux de mèche avec les hors la loi, les secteurs lucratifs de l'illégalité (prostitution et trafic de drogue), le curé mafieux, qui bénit à coups de goupillon les revolvers des narcotrafiquants, ou encore le marchand d'armes états-unien. Dans les deux cas, il s'agit bien de reconstituer une communauté à même d'évoquer, par la diversité et la représentativité de ses différentes composantes, la totalité du paysage national.

De là la réflexion sur le pouvoir qu'engagent les deux films, véritable fil conducteur de la cinématographie de Luis Estrada. L'objectif premier de ce questionnement semble devoir être de mettre en lumière la persistance des phénomènes de corruption du pouvoir au Mexique, du XX au XXI ${ }^{e}$ siècle. Cela se manifeste dans les deux œuvres étudiées par l'instauration d'une structure cyclique, fondée sur la circularité : en témoigne les scènes qui ouvrent et clôturent La ley de Herodes. La première revient sur les derniers instants de vie du maire de San Pedro de los Aguaros, qui, confronté à une insurrection populaire, se hâte de récupérer l'argent qu'il a accumulé durant son mandat et qu'il a caché un peu partout dans la mairie, avant de fuir et de se faire finalement tuer par les Indiens du village. Le reste du film, avec l'arrivée de Vargas comme nouveau maire, reproduit la dynamique qui, comme le spectateur l'imagine assez aisément, a conduit l'ancien dignitaire à cette fin tragique: Vargas s'enrichit peu à peu aux dépens du peuple, dissimule l'argent extorqué aux Indiens dans le trou qu'il a creusé au milieu d'un volumineux exemplaire de la Constitution (comme son prédécesseur), et finit par être sauvé in extremis d'une rébellion populaire, qui l'avait contraint à grimper en haut de 
l'unique poteau électrique du village. L'image est lourde d'ironie, tant la dimension christique qu'acquiert à cet instant le maire réfugié en haut d'un poteau aux allures de croix contraste avec l'immoralité dont a fait preuve le personnage tout au long du film. La scène finale corrobore la thèse d'une temporalité cyclique en présentant l'arrivée de son successeur à la mairie de San Pedro via une séquence qui reproduit au mot près l'arrivée de Vargas et de sa femme, quelques semaines plus tôt. Par ailleurs, alors que les événements du film se déroulent en 1949, sous la présidence de Miguel Alemán (1946-1952), plusieurs éléments établissent des ponts entre la situation mexicaine du milieu du XXe siècle et les années 1990, contemporaines de la réalisation du film, amplifiant ainsi la dynamique cyclique déjà présente dans la seule diégèse: pensons notamment à la rhétorique officielle utilisée par le régime de Miguel Alemán, dont le film se fait l'écho de manière insistante, en retranscrivant l'aspiration à la modernisation qui était au cœur des discours de l'époque. Le slogan grâce auquel avait été élu Miguel Alemán, "Modernidad y justicia social », est ainsi répété tout au long du film, et se matérialise de manière ironique par les efforts de Vargas tentant vainement d'apporter l'électricité à San Pedro. Or, les années 1940, sous les présidences de Ávila Camacho et Miguel Alemán, et les années 1990, notamment sous la présidence de Carlos Salinas de Gortari (1988-1994), ont en commun cette définition presque obsessionnelle du Mexique comme une nation moderne, en passe d'atteindre une situation économique caractéristique des pays dits "développés ", au premier desquels figurent les États-Unis. Conforté par la signature du traité de l'ALENA, entré en vigueur en 1994 (traité que pourraient incarner à l'écran les accords passés entre Vargas et Robert Smith), Carlos Salinas de Gortari se distingue effectivement par l'utilisation d'une rhétorique triomphaliste, qui l'amène à tenir des propos de ce type : «Avanzamos efectivamente en la estabilidad, abatiendo la inflación hasta niveles muy cercanos a los del mundo desarrollado " (Salinas de Gortari, cité par Concepción Montiel, 2002 : 214). De même, les complots agitant le champ politique, qui conduisent certains personnages de La ley de Herodes à faire assassiner leurs principaux rivaux, pourraient évoquer deux des plus célèbres meurtres des années 1990 au Mexique, à savoir Luis Donaldo Colosio, candidat du PRI à la présidence en 1994, et José Francisco Ruiz Massieu, député fédéral assassiné la même année. En l'espace d'un demi-siècle, donc, rien ne semble avoir entamé la dynamique de corruption dans lequel est engagée la politique mexicaine.

Le constat s'avère également valable dans le Mexique de El infierno, soit plus de dix ans après La ley de Herodes, comme l'attestent différents épisodes du film: pensons par exemple aux forces de police qui se laissent soudoyer par Benjamín, venu négocier la sortie de prison de son neveu, au point de lui dire finalement: "Ya se va acordando de cómo funcionan las cosas en México. » De même, la corruption des politiques est évoquée de diverses manières, parmi lesquelles la plus significative serait sans nul doute la présence, dans le bureau du chef de cartel de drogue local, don José, de photographies le montrant souriant aux côtés de Miguel de la Madrid, Carlos Salinas de Gortari, Ernesto Zedillo et Vicente Fox, soit les hommes ayant été à la tête de l'Etat de 1982 à 2006 . La frontière entre réalité est fiction est alors abolie, décuplant le potentiel transgressif du film. Par ailleurs, il est intéressant de constater que le parallèle hautement subversif entre politique et narcotrafic est accentué, pour tout spectateur familier de la filmographie d'Estrada, par le fait que le même acteur, Ernesto Gómez Cruz, joue respectivement d'un film à l'autre un influent gouverneur membre du PRI, aspirant à la chaire présidentielle, et un baron de la drogue. 
9 De la corruption, les deux films glissent finalement vers la représentation d'un pouvoir irrémédiablement lié à la mort, semblant ainsi confirmer les théories de Michel Foucault, pour qui «la vie et la mort ne sont pas de ces phénomènes naturels, immédiats, en quelque sorte originaires et radicaux, qui tomberaient hors $\mathrm{du}$ champ $\mathrm{du}$ pouvoir politique » (Foucault, $1997: 214)$. Dans l'œuvre d'Estrada, qu'il s'agisse de l'action politique à proprement parler, dans le cas de La ley de Herodes, ou du pouvoir au sens large du terme, dans El infierno, les instances incarnant l'autorité deviennent dans chaque œuvre agents de mort. Dans le film de 1999, une des premières responsabilités à laquelle est confrontée le nouveau maire, Vargas, est précisément d'enterrer un cadavre dont il a hérité et dont personne ne semble se préoccuper : symboliquement, son activité politique le lie ainsi dès son arrivée à la mort. Mais le film ne se contente pas de faire de Vargas une victime collatérale de la mort, l'obligeant ici à faire office d'employé de pompes funèbres : au contraire, le maire fraîchement nommé va comprendre rapidement que l'exercice du pouvoir implique de prendre part activement à la concrétisation de la mort. En témoigne l'attitude de son supérieur, qui lui donne pour seuls outils susceptibles de l'aider à administrer son village un exemplaire de la Constitution et un revolver, en ajoutant: « Con el librito y la pistola, a ejercer la autoridad. » Á partir de là, Vargas entreprend une opération d'élimination systématique de ses ennemis ou des personnes mettant en péril son exercice du pouvoir, ce qui l'amène à assassiner cinq personnes au cours de son bref mandat. Dans les films d'Estrada, la politique est ainsi une nécropolitique, ce qui semblerait faire du Mexique un de ces espaces où disparaîtrait le biopouvoir moderne, qui, d'après la terminologie foucaldienne, consiste à "faire vivre et laisser mourir » (Foucault, 1997:214), au profit d'un retour de l'ancien pouvoir souverain, dont les prérogatives étaient précisément de «faire mourir et laisser vivre ». Cela se vérifie également dans El infierno, via la persistance d'une réflexion sur la nécropolitique, les hommes politiques étant subventionnés par les narcotrafiquants, et ceux-ci jouissant en contrepartie de garanties d'impunité. Toutefois, ce second film élargit la perspective en sortant du seul champ politique, afin de souligner l'existence d'un nécropouvoir, en l'occurrence celui détenu par les narcotrafiquants. Modalité du pouvoir propre à ce Mexique du XXI ${ }^{\mathrm{e}}$ siècle (ou dont l'importance s'est pour le moins accrue à la charnière des deux siècles), le fonctionnement interne des cartels de drogue ainsi que le droit de vie et de mort dont ils disposent sur les membres de la société attestent finalement, au-delà de la sphère politique, de l'indissociabilité du pouvoir et de la nécessité de tuer.

10 Toutefois, si La ley de Herodes et El infierno posent tous deux la question de la nécropolitique et du nécropouvoir, le second film va plus loin dans ce questionnement sur la mort. Nous proposons ainsi d'appliquer à El infierno le concept de nécroesthétique, en partant du constat que le film se fonde sur une systématisation du recours à la mort comme métaphore d'un pays en crise. Cela passe dans un premier temps par l'élaboration d'un cadre spatial placé d'emblée sous les auspices de la mort. Rien d'anodin à ce que la première chose que voie Benjamín au moment de son retour à San Miguel soit le cadavre d'un homme venant tout juste d'être assassiné en pleine rue. De même, l'attitude des passants ayant assisté au meurtre, qui profitent des quelques secondes séparant la mort de l'homme de l'arrivée de la police pour dépouiller le cadavre, rappelle immanquablement les charognards que le spectateur attentif aura vu planer au-dessus d'une décharge publique, au début du film. D’emblée, la ville est donc présentée comme l'espace de la mort. À mesure qu'il avance dans la ville, Benjamín découvre un paysage désolé et envahi par les détritus, où les lieux de vie qu'il fréquentait avant son départ, tel 
que le garage de son parrain, sont maintenant laissés à l'abandon. Par ailleurs, toute tentative de recréer la vie semble illusoire: lorsque, plus tard dans le film, Benjamín décide de mettre à profit l'argent gagné grâce au narcotrafic pour aider son parrain à rebâtir un garage flambant neuf, qui apparaît comme un îlot de vie au milieu d'une zone désertique dont les routes ne semblent fréquentées que par les narcotrafiquants, la réaction de l'oncle, ébahi face à la construction, est significative : « Pues ahora nomás nos faltan los clientes. » Le narcotrafic, seule activité prospère de la région, ne propose ainsi qu'un semblant de vie, incapable de remédier à l'inertie ambiante.

11 De même, San Miguel est placé sous le signe de la fatalité, qui fait de la mort à court ou moyen terme le seul horizon possible pour ses habitants. La représentation de la jeunesse est particulièrement révélatrice à cet égard: mimétisant les comportements des narcotrafiquants adultes, les jeunes érigent ces derniers en figures héroïques dans les pas desquels il convient de s'inscrire. Cela passe notamment par l'exaltation de la violence et l'utilisation d'une rhétorique machiste faisant de l'homme aussi habile dans le maniement des armes que dans ses relations avec les femmes un modèle de virilité, et donc, pour ces jeunes, un modèle tout court. Pensons par exemple à la fierté éprouvée par le neveu de Benjamín à l'égard de son défunt père, un ancien narcotrafiquant que le jeune homme de quatorze ans décrit en ces termes: «Mi jefe era el más chingón de este pinche pueblo, dicen que era bien cabrón con las viejas pero mejor con las pistolas. Si hasta dicen que mató a más de veinte cabrones. » Très logiquement, lorsque Benjamín lui demande ce qu'il souhaite faire une fois adulte, son neveu lui répond alors : «Pues qué otra cosa, pues un chingón como mi papá.» L'avenir lui donnera raison: alors que le jeune homme semblait représenter la seule note d'espoir du film, après être parvenu à s'extraire de San Miguel et à gagner les États-Unis, le film s'achève sur son retour au pays, alors qu'il est venu venger la mort de toute sa famille en assassinant les nouveaux barons du trafic de drogue local. L'image se fige sur cette vision du jeune homme, la mitraillette à la main et le visage déformé par la rage, attestant de l'incapacité du pays à s'extraire de la dynamique infernale dans laquelle il s'est engagé. Ce plan final s'inscrit par ailleurs dans le prolongement d'une séquence dans laquelle les bandes d'adolescents de San Miguel s'entraînent à manier le pistolet et à viser en tirant sur des poules, ou encore à viser à distance des véhicules qu'ils ne peuvent atteindre. Ces attitudes sont la preuve d'une violence latente et d'un pouvoir mortifère en devenir, dont la séquence finale marque la concrétisation. Dès lors, les habitants de San Miguel, qu'il s'agisse d'adolescents ou d'adultes, vivent avec la pleine conscience de l'omniprésence et de l'inévitabilité de la mort : en témoignent les paroles de Cochiloco, le meilleur ami de Benjamín, qui explique que la mort fait partie du quotidien pour les narcotrafiquants, ou encore, de manière plus flagrante encore, les mots du neveu de Benjamín, qui demande à son oncle si celui-ci pourra surmonter sa tombe d'un mausolée lorsqu'« on le tuera ", à condition, ajoute-t-il, que son oncle soit lui-même encore en vie.

12 Mais l'apparition de la mort ne concerne pas la seule sphère du langage, et le cadavre sur lequel tombe Benjamín en entrant dans San Miguel n'est en réalité que le prélude au déferlement de violence auquel va assister le spectateur tout au long du film. La mort envahit véritablement l'écran, l'insistance voire l'obsession pour la représentation de la mort étant sans commune mesure avec ce qui se produisait onze ans plus tôt, dans La ley de Herodes. Sur le plan purement quantitatif, on peut dénombrer entre quarante et cinquante morts dans $E l$ infierno, qu'elles apparaissent via la simple évocation, le meurtre se produisant hors champ, ou que celui-ci soit au contraire montré à l'écran, le film 
n'étant pas avare de ces scènes d'exécution. La représentation de la mort se fait par ailleurs plus présente au cours de la seconde moitié du film, reproduisant en cela le processus d'initiation vécu par le héros, Benjamín García, depuis la découverte horrifiée des procédés barbares propres à l'univers du narcotrafic jusqu'à sa pleine participation à ces mêmes pratiques. Le style adopté par Luis Estrada se caractérise par une extrême crudité et une tendance à emprunter aux codes de l'esthétique gore, les narcotrafiquants rivalisant d'inventivité au moment d'exécuter leurs rivaux: exécutions à bout portant, fusillades, multiples décapitations, oreilles arrachées, lèvres tranchées au machete, mains coupées à la tronçonneuse, corps démembrés puis dissous dans de l'acide, sans oublier les inévitables et incessantes effusions de sang, le réalisateur semblant par exemple avoir un goût prononcé pour l'image du sang jaillissant du crâne. L'impact visuel de ces scènes est par ailleurs amplifié par l'utilisation de plans resserrés, notamment s'agissant des têtes arrachées au corps, ou encore par la récurrence de certains plans suggérant l'indissociabilité de l'homme et de l'arme. Ainsi, on soulignera l'importance des plans de profil, alors que le personnage est sur le point de tuer. Outre le fait qu'ils permettent dans certains cas d'apprécier la faible distance qui sépare le bourreau de sa victime, donc de souligner l'extrême violence de l'acte, ces plans de profil font du revolver un prolongement naturel du bras tendu du personnage, comme si la faculté à tuer lui était intrinsèque. Signalons toutefois que, ce déferlement de violence s'inscrivant dans le cadre d'une comédie, la mort bénéficie d'un traitement qui inspire le sourire, voire le franc éclat de rire, davantage que l'effroi. Toute une série d'éléments viennent en effet prendre le contrepied de la dimension tragique et/ou terrifiante qui pourrait accompagner la mort : un procédé parmi les plus récurrents, déjà présent dans La ley de Herodes, consiste notamment à contrebalancer l'horreur visuelle par une bande son aux accents joyeux, par exemple via l'utilisation de la musique norteña, qui instaure un effet de contraste particulièrement saisissant entre les tonalités festives de l'accordéon et l'extrême violence des images projetées simultanément à l'écran. De même, l'escalade de l'horreur en vient parfois à provoquer des effets comiques inattendus, par exemple lorsque les narcotrafiquants confirmés restent pantois face à la brutalité presque animale des nouvelles recrues, qui surpassent leurs maîtres en cruauté et se moquent de la pusillanimité de ces derniers.

13 Par ailleurs, une des composantes de la nécroesthétique adoptée dans El infierno serait le lien tissé entre la réalité géographique représentée et le thème de l'Enfer, espace des morts par excellence, présent dès le titre. Notons que les premières secondes du film donnent le ton, puisque le titre apparait à l'écran via une graphie rouge stéréotypée semblant annoncer un film d'horreur. L'espace mexicain s'impose en effet dans El infierno comme une forme de pandémonium moderne, et ce à différents niveaux. La manifestation la plus évidente de la métaphore infernale provient, dans un premier temps, des multiples maux qui semblent s'être abattus sur le village natal de Benjamín García. Le panorama qui s'offre aux yeux du spectateur se distingue par sa noirceur extrême: lorsqu'il rentre des États-Unis, le héros du film découvre effectivement un monde de désolation et de saleté, un monde où la violence et la corruption règnent en maîtres. Dès son retour, lors des retrouvailles de Benjamín et de son parrain, celui-ci le met en garde : «Tu ne peux pas imaginer ce qu'est devenu ce pays» lui dit-il, confirmant la une de journal que lit au même moment Benjamín et qui titre "Crise et violence dévastent le pays ». Ces avertissements initiaux se confirment par la suite lorsque le héros fait l'expérience en chair propre d'une réalité marquée au sceau de l'horreur et de l'injustice : corruption, blanchiment d'argent, trafic d'armes et de drogue, délation, menaces, 
agressions, braquages, assassinats, voilà autant de phénomènes constituant le quotidien du village frontalier décrit dans le film.

Le cinéaste brosse ainsi un portrait extrêmement sombre de la réalité mexicaine, ce qui, en soi, suffirait à ranger Luis Estrada dans la catégorie des artistes assumant le risque d'affronter un véritable "procès en mexicanité » (Amiot, 2011: 56), c'est-à-dire dans le clan de ceux qui choisissent délibérément de s'éloigner d'une description tout à la gloire de l'espace national. La tentation est grande de comparer l'intention d'Estrada à celle qui animait Luis Buñuel au moment où il réalisa un film ayant provoqué un scandale aussi retentissant au Mexique que Los Olvidados (1950). Dans ce film, Buñuel, qui venait d'obtenir la nationalité mexicaine un an auparavant, décrit son pays d'adoption sous un jour éminemment transgressif, en choisissant de braquer sa caméra sur la réalité sordide des gamins de banlieues défavorisées. Bien loin des stéréotypes traditionnels véhiculés par le cinéma mexicain de l'époque, qui s'en tenait à une vision édulcorée et idéalisée de la réalité du pays, le film entre en contradiction évidente avec l'image officielle défendue par l'Etat. Comme l'écrit Julie Amiot, « [d]ans une période au cours de laquelle le Mexique souhaite donner de lui-même l'image d'une nation moderne, industrialisée, urbaine et sociale, bref, tournant résolument le dos au "sous-développement", le film pouvait à juste titre apparaitre comme une provocation et courait le risque de s'attirer les foudres d'un État au discours duquel il apportait un terrible démenti » (ibid. : p. 41-42). Avec El infierno, Luis Estrada s'inscrit à notre avis dans le prolongement direct de Buñuel en s'engageant dans une entreprise de démystification voire de démythification, alors même que le film est censé participer aux cérémonies de célébration du bicentenaire de l'Indépendance. En mettant en lumière l'enfer quotidien que signifie pour les habitant du village la vie à San Miguel, le film d'Estrada se situe aux antipodes d'une opération de glorification et montre au contraire, comme l'indique l'affiche du film, qu'il n'y a « rien à célébrer ».

Au-delà de la seule noirceur de l'univers dépeint dans le film, le parallèle avec le motif de l'Enfer se justifie par des allusions plus précises, voire même parfois explicites, à la symbolique qui entoure, dans l'imaginaire biblique, le domaine de Satan. L'association de l'espace mexicain et de l'Enfer débute dès le début du film. Les deux premières séquences, entre lesquelles se sont écoulés vingt ans, donnent respectivement à voir le départ aux États-Unis, puis le retour de Benjamín García au Mexique. La séquence qui ouvre le film nous présente trois personnages, à savoir le héros, sa mère et son frère cadet, au moment de la séparation: l'intérêt de la scène provient notamment de l'effet de silhouettage choisi par le cinéaste, effet qui, par le biais de l'éclairage en contre-jour, permet de souligner les contours des trois figures se découpant sur un arrière-plan lumineux. La toile de fond, auréolée d'un soleil naissant, renvoie métaphoriquement à la frontière avec les États-Unis, vers laquelle se dirige Benjamín : si l'association de la lumière et de l'espoir que constitue pour le héros ce départ vers de nouveaux horizons est évidente, il n'en est pas moins intéressant de constater que dès les premières secondes du film, le Mexique est au contraire assimilé à l'ombre. Confirmant ainsi un titre déjà évocateur, le symbolisme de la lumière nous oriente vers une lecture manichéenne qui associerait les États-Unis à la lumière et le Mexique au règne de l'obscurité, ce que corroborera la suite du film. Cette scène n'est pas sans rappeler un des procédés rhétoriques et symboliques les plus fréquemment utilisés dans les Saintes Écritures pour rendre compte de l'opposition entre le Bien et le Mal. Si les ténèbres sont dans la Bible porteuses de malheur et de mort, donc liées à Satan, la lumière y est au contraire la marque du divin : à la fois en tant que source et principe de toute vie sur Terre, comme en témoigne la première parole prononcée par 
Dieu dans la Genèse ("Que la lumière soit ! »), et en tant que métaphore de l'être divin à proprement parler. Parmi les multiples occurrences faisant de la lumière un élément symptomatique du divin, Jésus-Christ dit par exemple aux apôtres dans l'Evangile selon Jean : " Je suis la lumière du monde. Celui qui vient à ma suite ne marchera pas dans les ténèbres ; il aura la lumière qui conduit à la vie » (Nouveau Testament, Jean, 8, 12). Le lien avec l'imaginaire chrétien est d'autant plus justifié que le recours à l'intertexte biblique est omniprésent dans le film, bien qu'à des fins satiriques.

La seconde séquence du film, véritable contrepoint de la première, nous présente au contraire Benjamín au poste de frontière, menotté et sur le point d'être libéré pour rentrer au Mexique. Le plan d'ensemble qui ouvre la séquence permet au spectateur d'apprécier les différents éléments matérialisant la sensation désagréable qui accompagne le retour forcé du clandestin dans son pays d'origine : barbelés, grillages, murs, autant de détails marquant une frontière nette entre l'espace fantasmé des ÉtatsUnis, qui ne sera justement jamais montré dans le film, et l'espace mexicain. Le point de convergence des deux scènes évoquées, bien que celles-ci soient diamétralement opposées, serait de créer un effet de suspense en retardant le dévoilement du lieu annoncé métaphoriquement par le titre et qui constituera le cœur même du film, le Mexique. Dans le premier cas, l'espace mexicain reste plongé dans l'ombre, tandis que lors du retour de Benjamín, le réalisateur choisit précisément de ne pas montrer la destination de l'immigré mais le lieu d'où il vient. Dans les deux scènes, ainsi, on cache volontairement l'espace qui est rejeté et que l'on cherche à fuir. Notons également l'ironie qui accompagne ce retour au Mexique, comme l'atteste la phrase lancée par le policier à celui qui vient de se faire expulser des États-Unis, "Welcome back to Mexico ", immédiatement suivie d'un très sec « Don't come back ». La bande son va alors prendre le relais de cette ironie en créant un effet de contraste, né de la juxtaposition d'une musique norteña et d'images décrivant le retour cauchemardesque de Benjamín au pays. Un long travelling latéral permet en effet de reproduire le triste panorama qui s'offre aux yeux du héros depuis la fenêtre du bus, et qui voit se succéder des paysages arides et désertiques, des déchetteries survolées par des charognards, ainsi qu'un autel dédié au culte de la Santa Muerte. Rappelons que ce culte condamné par l'Eglise, qui s'est essentiellement développé dans la seconde moitié du XXe siècle au Mexique, notamment dans les zones défavorisées, est une forme de syncrétisme mêlant éléments catholiques et croyances préhispaniques. Prônant la dévotion à la Mort, incarnée sous les traits d'un squelette habillée en femme, le culte de la Santa Muerte est souvent associé, dans l'imaginaire collectif, aux milieux délictueux, notamment à l'univers du trafic de drogue. Dès le départ, donc, le retour à l'espace mexicain est associé soit à la décrépitude, soit à la mort. Par ailleurs, alors que résonne toujours dans l'oreille du spectateur les accents joyeux de la musique norteña qui a débuté avec le voyage en bus, Benjamín est victime de deux agressions, se faisant successivement dépouiller par des braqueurs et par des policiers. Enfin, signalons que le trajet du héros s'accompagne d'un retour à l'obscurité, alors qu'il entre dans la maison familiale plongée dans la pénombre : le parallèle est alors inévitable avec l'ombre dans laquelle se trouvait déjà l'espace mexicain vingt ans plus tôt, lors de son départ pour les États-Unis. Qu'il s'agisse donc des paysages montrés à l'écran, de la violence qui accompagne le retour au Mexique, ou encore de l'utilisation métaphorique de la lumière, tout concourt à fixer l'impression que le territoire mexicain correspond bien à l'Enfer évoqué par le titre du film. 

San Miguel Arcángel. L'archange Saint Michel étant précisément le chevalier ayant, selon la tradition, terrassé le Diable, on voit poindre le détournement parodique de la symbolique biblique auquel s'adonne Luis Estrada dans le tout le film: le village de San Miguel Arcángel y est effectivement présenté comme le point névralgique du trafic de drogue de la région, contrôlé par les deux cartels que dirigent les frères Reyes. De là la lettre « $\mathrm{N}$ » qui a été ajoutée à la peinture noire sur la pancarte à l'entrée de la ville, qui transforme « Arcángel » en «Narcángel » et renvoie, par jeu d'homophonie, à l'univers des narcotrafiquants. En outre, Benjamín découvre rapidement que le frère qu'il a laissé vingt ans plus tôt, qui s'est fait entre-temps assassiner, était devenu un des trafiquants les plus redoutés de San Miguel, au point d'être surnommé El Diablo. Au bout du compte, le village qui semblait être placé sous la protection du divin, via la mention à l'archange Saint Michel, devient en réalité le terrain de jeu d'un personnage surnommé « Le Diable », donc directement associé à l'Enfer. San Miguel est également le lieu du vice, tant par l'omniprésence du trafic de drogue que par l'accent mis sur la description de la zona roja, la zone dédiée à la prostitution. Dans la lutte opposant Saint Michel au Démon, toute entière incarnée par le microcosme de San Miguel, c'est finalement ce dernier qui semble l'emporter : même mort, le frère de Benjamín, alias El Diablo, constitue en effet l'axe de la diégèse, autour duquel gravitent les principaux événements racontés dans le film. C'est ainsi la figure infernale qui structure l'œuvre dans son intégralité : à titre d'exemple, c'est pour honorer la mémoire de son frère que Benjamín décide de venir en aide à sa veuve et à son fils, donc d'intégrer le milieu du narcotrafic. C'est également le désir de vengeance de ce même fils, qui a découvert les origines troubles de la mort de son père, tué par les membres de son propre cartel de drogue, qui précipite la fin des événements et provoque le bain de sang final. Enfin, la meilleure illustration de la victoire du Diable serait sans nul doute la temporalité cyclique déjà mentionnée, qui fait de l'Enfer et de la mort le seul horizon possible pour le Mexique. En témoigne la perpétuation du nom du père au fils, le neveu de Benjamín étant précisément surnommé El Diablito en hommage à son père ; ce sont finalement les dernières secondes du film qui confirmeront le caractère héréditaire de la faculté à tuer, en évoquant les pulsions vengeresses du jeune homme.

En définitive, la perpétuation de ces schémas de violence et de mort participe de la dimension transgressive d'un film décrivant un Mexique en état de crise profonde, incapable de mettre fin aux maux qui le rongent. La mort y est partout, et, surtout, elle apparaît comme inhérente à la réalité mexicaine. C'est sans doute le sens qu'il faut donner à l'avant-dernière séquence de $E l$ infierno: Benjamín, qui n'a plus rien à perdre après que la femme qu'il aimait a été tuée, se rend sur la place de la ville au moment de la célébration du grito du 15 septembre, qui commémore le fameux grito de Dolores, et abat froidement tous ceux qui personnifiaient dans le film la corruption et la violence : anciens chefs de cartel, policier et maire corrompus, curé mafieux, etc. Dans le dernier plan, hautement symbolique, la caméra se fixe sur don José, le chef du cartel devenu maire, assassiné, dont le sang s'écoule lentement sur l'emblème du pays représenté sur le pupitre, l'aigle et le serpent. L'image est claire, évoquant un pays meurtri, et l'ironie particulièrement mordante, puisqu'au même moment les feux d'artifice de la fête continuent d'éclater et que s'affiche en lettres de feu le message "Viva México ». En systématisant ainsi le recours à la mort et en le liant de manière aussi flagrante à l'identité même de la nation qu'il était censé célébrer, Luis Estrada place le spectateur mexicain face à ses propres contradictions et face au constat amer contenu dans la 
fameuse «ley de Herodes ", qui semble résumer à elle seule le sort de l'homo mexicanus moderne : « o te chingas o te jodes ».

\section{BIBLIOGRAPHIE}

Amiot, Julie, "Reflets d'une société en mutation : le Mexique au milieu du XX ${ }^{\mathrm{e}}$ siècle ", in Amiot, Julie, et Alonso Carballes, Jesús, Le Mexique au milieu du XX $X^{e}$ siècle - La Transition démocratique en Espagne, Paris : Atlande, 2011.

Foucault, Michel, «Il faut défendre la société ». Cours au Collège de France (1975-1976), Paris : Seuil / Gallimard, 1997.

Rivera Garza, Cristina, Dolerse. Textos desde un país herido, Oaxaca : Sur+ / Frontera Press, 2011. Rivera Garza, Cristina, Los muertos indóciles. Necroescrituras y desapropiación, Mexico : Tusquets, 2013.

Salinas de Gortari, Carlos, Sexto informe de gobierno, cité par Concepción Montiel, Luis Enrique, El discurso presidencial en México : el sexenio de Carlos Salinas de Gortari, Madrid : Universidad Complutense de Madrid, 2002.

\section{Filmografie}

Estrada, Luis (réal.), El infierno, Mexique : Bandidos Films, 2010.

Estrada, Luis (réal.), La ley de Herodes, Mexique : Videomax, 1999.

\section{NOTES}

1. Morales reproche notamment à Vargas d'avoir autorisé le maintien au sein du village du bordel de Doña Lupe.

\section{RÉSUMÉS}

L'article se propose d'étudier la filmographie du réalisateur mexicain Luis Estrada, et plus particulièrement deux de ses œuvres, La ley de Herodes (1999) et El infierno (2010), à l'aune des concepts de nécropolitique et nécroesthétique. Il s'agira de déterminer comment la réflexion éminemment transgressive engagée par l'artiste sur le Mexique contemporain se nourrit du constat de l'indissociabilité du pouvoir et de la mort. A partir de là, l'œuvre s'engage sur une voie nouvelle, ne consistant plus seulement à lier le champ du pouvoir à la mort, mais à systématiser le recours esthétique à la mort comme métaphore d'un pays en crise. 
This article aims at ascertaining to what extent the filmography of the Mexican director Luis Estrada can be studied in the light of the concepts of necropolitic and necroesthetic, and will dwell more specifically on two of his works : La ley de Herodes (1999) and El infierno (2010). Our goal is to determine how the extremely transgressive reflection started by the artist about the contemporary Mexico derives from the assessment that power and death are indivisible. Based on this, the work steps into a new path and associates the concept of power with death, as well as it systematises the esthetic recourse to death as a metaphor of a country in a state of crisis.

INDEX

Mots-clés : Luis Estrada, cinéma, nécroesthétique, nécropolitique

Index géographique : Amérique Latine, Mexique

Keywords : Mexico, necroesthetic, necropolitic

\section{AUTEUR}

DAVY DESMAS

CUFR Champollion - Université de Toulouse

davy.desmas@gmail.com 